\title{
Optimized Solutions for the Arrangement of Digital Imaging Detectors
}

\author{
T. Hashimoto ${ }^{1}$, J. F. Mancuso ${ }^{2}$, K. Nakano ${ }^{3}$, E. Nakazawa ${ }^{1}$, L. Blubaugh ${ }^{4}$ and B. L. Armbruster ${ }^{5}$ \\ ${ }^{1}$ Hitachi High-Technologies Corporation, Ibaraki 312-8504, Japan \\ ${ }^{2}$ Advanced Microscopy Techniques Corporation, 242 W. Cummings Park, Woburn MA, USA \\ ${ }^{3}$ Hitachi High-Technologies Corporation, Kanagawa 213-0012, Japan \\ ${ }^{4}$ Hitachi High Technologies America, Inc., 22610 Gateway Center Dr., Clarksburg MD USA \\ ${ }^{5}$ Hitachi High Technologies America, Inc., 5960 Inglewood Dr., Pleasanton CA, USA
}

The Hitachi HT7700 120kV TEM is based on a product concept that allows all observation and image recording procedures to be done in daylight with fully digitized image acquisition devices [1]. A wideangle 1 megapixel camera monitors at 15 fps a fluorescent screen in an observation chamber positioned just below the projector lens. The live images are displayed in a window within Hitachi's system control GUI. A second window displays the image from the main image recording camera, imaging from a phosphor located in a traditional bottom-mount configuration. This camera can be an 8 megapixel CCD camera, XR81-B, or an optional high-sensitivity 4 megapixel scientific CMOS camera, XR401L-B. The bottom-mount camera is used for image recording and for automation functions [2], such as auto-focusing, stigmation and alignment, drift correction, stage translation and image acquisition for montaging, and sequential specimen tilting and image acquisition for electron tomography. Excellent high contrast at low magnification with a wide field of view (FOV) is also crucial to identify features of interest in correlative light/electron microscopy experiments [3].

AMT's XR16-DIR (Figure 1) was developed to address specific application fields such as pathology, histology, or anatomy that can require both a wider FOV than provided by conventional bottommounted cameras and finer image definition. The XR16-DIR camera exploits a 16M pixel CCD and a custom-made, finite conjugate lens to utilize a 41 by $62 \mathrm{~mm}^{2}$ area of the CCD scintillator, which is optimized for high contrast observation at $80 \mathrm{kV}$. Figure 2 depicts the camera layout on the TEM column. To ensure a wider FOV, the XR16-DIR is mounted directly under the HT7700 viewing chamber, "Flange (1)" as shown in Figure 2. Calculated practical image magnifications for the camera are about $60 \%$ of nominal magnifications displayed on the HT7700 monitor. The estimated image size of the XR16-DIR camera for the HT7700 at 500 times nominal magnification is about $140 \times 210 \mu \mathrm{m}$ on the specimen. It is almost $3 \times 3$ times wider than the area taken with a standard XR81-B camera single frame image and equivalent to the FOV of a $3 \frac{1}{4} \times$ x 4 " sheet of photographic film. Due to the larger number of pixels, the frame rate without binning is slower than that of the XR81-B. Thus auto focusing must be done with binned sub-areas. Such operational conditions are necessary to obtain higher quality images with a reduced noise component. Finally, in addition to a greatly expanded FOV, viewers experience brighter images for a given set of column conditions. Usability improves for application fields such as renal pathology, as exemplified in Figure 3.

\section{References:}

[1] H. Tanaka et al., Proc. $67^{\text {th }}$ Annual Meeting of the Japanese Society of Microscopy, 16Apm_I1-3 (2011) p.8

[2] T. Hashimoto et al., Proceedings M\&M2012, Phoenix (2012) p. 1280.

[3] W.G. Janssen, H.H. Hanson and B.L. Armbruster, Proceedings M\&M2014, Hartford (2014) p. 1104. 


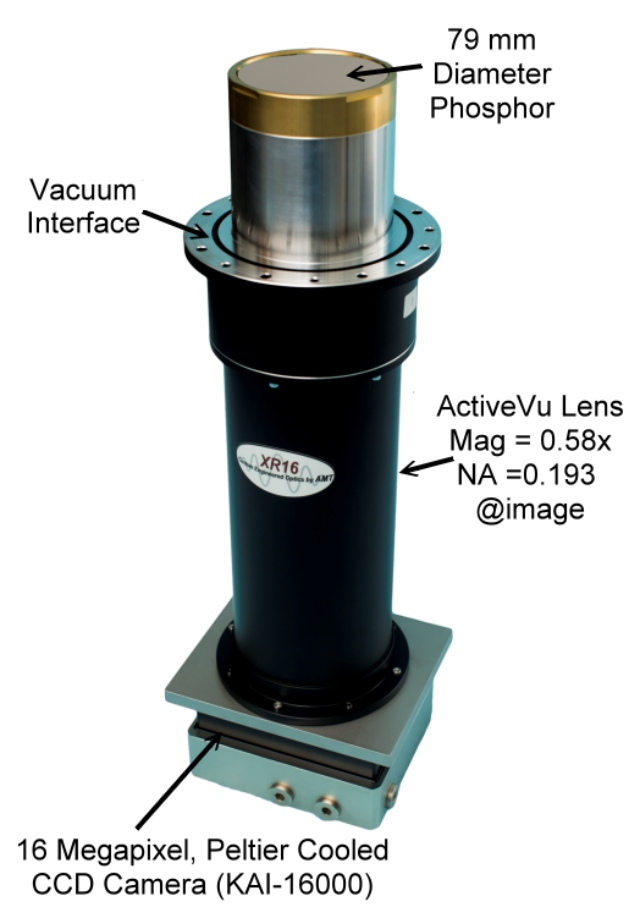

Figure 1. XR16-DIR camera configured to mount to the HT7700 column at Flange (1).

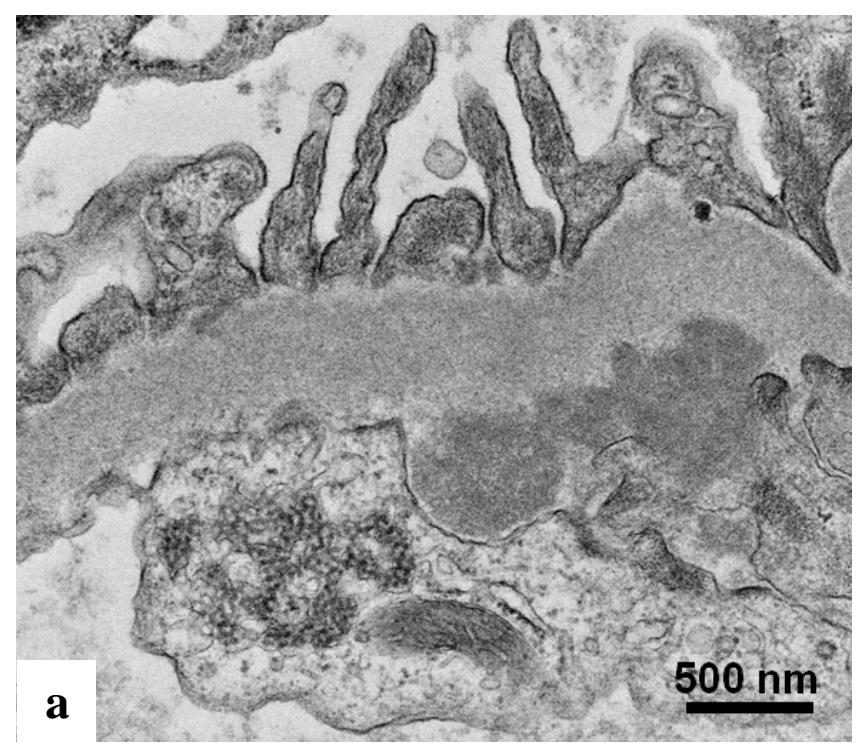

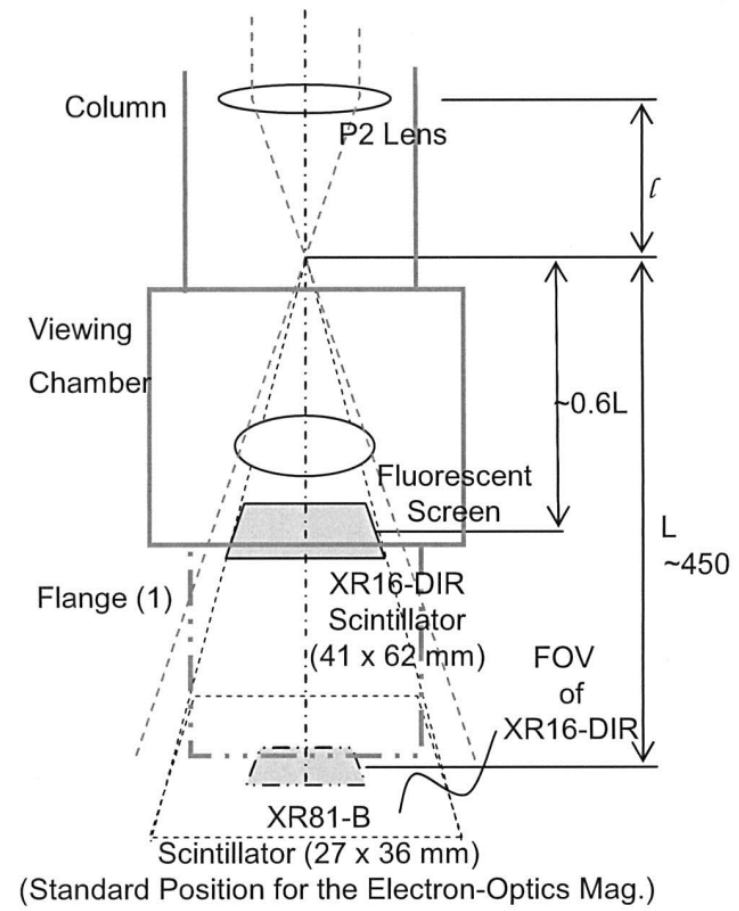

Figure 2. A FOV comparison of XR16-DIR and XR81-B cameras at different column positions.

\section{AMT XR16-DIR Specification}

Number of pixels...............3,248 $\times 4,864$ pixels Frame rate................. 8 frame $/ \mathrm{s}$ ( $4 \times 4$ binning) FOV range........41.44 $\times 62.06 \mathrm{~mm}$ (on scintillator) Pixel size on CCD...................... $7.4 \times 7.4 \mu \mathrm{m}$ Pixel size on specimen at $10 \mathrm{k} \times \ldots . .2 .111 \times 2.111 \mathrm{~nm}$ (Estimated, $1.083 \times 1.083 \mathrm{~nm}$ if camera is XR81-B) Coupling............................... Optical lens Scintillator............Phosphor optimized for $80 \mathrm{kV}$

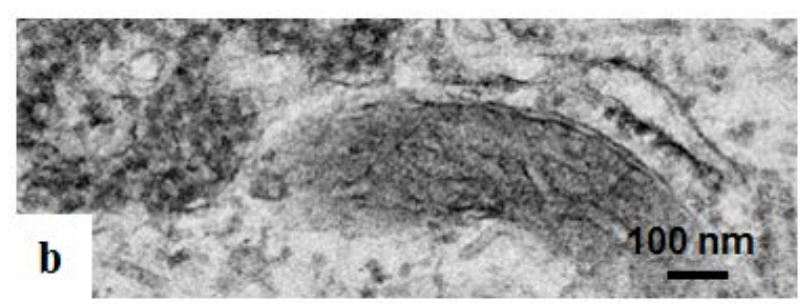

Figure 3. a) Characteristic ultrastructural features of Lupus nephritis including subendothelial deposits and tuboreticular inclusions were imaged on the HT7700 by means of the XR16-DIR camera, b) a subarea of (a) enlarged 4 times. Main specifications for the XR16-DIR camera are included above. 